revised for Computer Physics Communications, July 6, 2016

\title{
Computational Thermochemistry: Automated Generation of Scale Factors for Vibrational Frequencies Calculated by Electronic Structure Model Chemistries
}

Haoyu S. Yu, Lucas J. Fiedler, I. M. Alecu, and Donald G. Truhlar*

\begin{abstract}
We present a Python program, Freqscale, for calculating the optimal scale factors for calculating harmonic vibrational frequencies, fundamental vibrational frequencies, and zeropoint vibrational energies from electronic structure calculations. The program utilizes a previously published scale factor optimization model [I. M. Alecu, J. Zheng, Y. Zhao, D. G. Truhlar, J. Chem. Theory Comput. 6 (2010) 2872] to efficiently obtain all three scale factors from a set of computed vibrational harmonic frequencies. In order to obtain the three scale factors, the user only needs to provide zero point energies of 15 or 6 selected molecules. If the user has access to the Gaussian09 or Gaussian03 program, we provide the option for the user to run the program by entering the keywords for a certain method and basis set in the Gaussian09 or Gaussian03 program. Four other Python programs, input.py, input6, pbs.py, and pbs6.py are also provided for generating Gaussian 09 or Gaussian 03 input and pbs files. The program can also be used with data from any other electronic structure package. A manual of how to use this program is included in the code package.
\end{abstract}




\section{Introduction}

Computational electronic structure theory is used to predict many useful properties of molecules. The harmonic vibrational frequencies obtained from such quantum chemical computations are widely employed to estimate spectroscopic, thermochemical, and dynamical properties of molecules. At the molecular level, computed harmonic vibrational frequencies can be used to approximate the spectroscopic energy differences associated with energetic transitions between various vibrational states of a molecule as well as to provide a measure of the molecule's vibrational zero-point energy (ZPE). In addition, within the harmonic oscillator approximation, the molecular vibrational partition function, which is the fundamental quantity underlying molecular statistical mechanics for vibrational degrees of freedom and which links molecular properties to bulk thermochemical properties, can be conveniently estimated via a simple analytical expression utilizing the computed vibrational frequencies; these partition functions can then be used for a variety of calculations of thermochemistry and thermochemical kinetics.

The harmonic approximation is very practical because it only requires the quadratic force constants, which can be obtained from electronic structure calculations in the Born-Oppenheimer approximation. Although the practicality of the harmonic oscillator approximation has made this method very popular for computing vibrational frequencies, vibrational properties derived from computed harmonic vibrational frequencies can often disagree considerably with their experimentally-determined counterparts. This discrepancy between computed and observed vibrational properties has two origins. The first is the neglect of anharmonicity in the harmonic oscillator approximation. The second is the deviation of the electronic structure treatment from complete configuration interaction, which would correspond to the exact solution of the electronic Schrödinger equation. The deviation from complete configuration interaction is due to the incomplete treatment of electron correlation in the level of theory as well as the incompleteness of the one-electron basis set used to expand the orbitals used to construct configuration state functions. The errors resulting from these effects are usually found to be systematic. The error due to anharmonicity can be minimized either by calculating and applying anharmonic corrections or - at least for the higher frequencies - by scaling the computed harmonic frequencies, and the error due to incompleteness of the electronic structure calculation 
can be partially overcome by selecting a level of theory that accounts well for electron correlation and pairing it with a well-balanced one-electron basis set. Systematic progress on the latter aspect is possible by identifying theoretical model chemistries, [1] which are well-defined general methods, such as the combination of Hartree-Fock theory or a particular Kohn-Sham exchange-correlation functional with a particular basis set. From this point onward, building on the language of Pople,[1] we will denote a combination of a level of theory (or choice of density functional) and a one-electron basis set as an electronic model chemistry.

In addition to the quadratic force constants needed in the computation of harmonic frequencies, the evaluation of anharmonic corrections also requires higher-order (e.g., cubic and quartic) force constants. The calculation of vibrational energy levels by including higher force constants can be accomplished by perturbative approaches $[2,3,4,5,6,7,8]$ or by variational methods $[9,10,11,12,13,14,15,16,17,18,19]$. However, the higher-order force constants required as input for such calcuations are expensive to compute. Consequently, the procedure of scaling the computed harmonic vibrational frequencies to compensate for the neglect of anharmonicity has become commonplace, and scale factors have been devised for numerous electronic model chemistries. In fact, a recent study[20] has shown that it is beneficial to scale even the anharmonic vibrational frequencies obtained from second-order perturbation theory.

As previously explained,[21,22,23] scale factors are property-specific, and in the past, they have been predominantly optimized to accurately reproduce true harmonic vibrational frequencies, observed fundamental vibrational frequencies, and/or vibrational ZPEs; although, scale factors customized to better reproduce vibrational contributions to various thermodynamic quantities have also been proposed.[22] Although applying scale factors to correct for the systematic errors that arise from using harmonic vibrational frequencies to estimate vibrational properties of interest is convenient, the optimization of such scale factors can be a tedious and time-consuming process, especially if scale factors are needed for several electronic model chemistries and for more than one property. In a recent article,[23] we introduced a practical method for efficiently evaluating the optimal scale factors for reproducing the true harmonic vibrational frequencies, fundamental vibrational frequencies, and ZPEs from a set of computed harmonic vibrational frequencies. The subject of the present article is to introduce and describe 
FREQ, a program that further increases the efficiency of scale factor optimization through automation.

\section{The Scale Factor Optimization Process}

The use of harmonic oscillator formulas with effective frequencies is called the quasiharmonic approximation. Scale factors are empirically-derived factors to be applied to computed harmonic frequencies in order to improve the accuracy of properties being modeled by the quasiharmonic approximation. For a given electronic model chemistry, the first step toward deriving the scale factor for the property of interest is computing this property for a set of molecules for which this property is accurately known from either experiments or high-level calculations. Then, operating under the assumption that the computed and actual properties are proportional to one another, it is customary to equate the scale factor (which we will call $\lambda$ ) to the optimal value for the proportionality constant, in particular the value that minimizes the root mean squared (RMS) deviation between the computed and accurate data sets. In previous work from our group, scale factors for harmonic vibrational frequencies were optimized against the 38 accurate harmonic frequencies compiled in the F38/06 database,[24] scale factors for fundamental vibrational frequencies were optimized to best reproduce the 38 accurate fundamental frequencies in the F38/10 database,[23] and scale factors for ZPEs were optimized to best reproduce the ZPVE13/99,[25] ZPVE15/06,[24] and ZPVE15/10[23] databases consisting of 13,15 , and 15 ZPEs, respectively.

Obtaining three property-specific scale factors for even one electronic model chemistry by fitting computed data against such extensive databases is already a laborious task, and if scale factors are needed for a multitude of electronic model chemistries, optimizing these can quickly become extensively time-consuming and computationally intensive. We therefore proposed[23] a scale factor optimization model to obtain the scale factors for true harmonic frequencies $\left(\lambda^{\mathrm{H}}\right)$, fundamental frequencies $\left(\lambda^{\mathrm{F}}\right)$, and ZPEs $\left(\lambda^{\mathrm{ZPE}}\right)$ for a given electronic model chemistry from a set of harmonic frequencies computed with that same electronic model chemistry. Specifically, this model entails explicitly deriving just $\lambda^{\mathrm{ZPE}}$, and then obtaining $\lambda^{\mathrm{H}}$ and $\lambda^{\mathrm{F}}$ through the relations

$$
\lambda^{\mathrm{H}}=\alpha^{\mathrm{H} / \mathrm{ZPE}} \lambda^{\mathrm{ZPE}}
$$




$$
\lambda^{\mathrm{F}}=\alpha^{\mathrm{F} / \mathrm{ZPE}} \lambda^{\mathrm{ZPE}}
$$

where the proportionality constants $\alpha^{\mathrm{H} / \mathrm{ZPE}}$ and $\alpha^{\mathrm{F} / \mathrm{ZPE}}$ are called the universal scale factor ratios[23] in light of the fact that they should be independent of the electronic model chemistry. The values of $\alpha^{\mathrm{H} / \mathrm{ZPE}}$ and $\alpha^{\mathrm{F} / \mathrm{ZPE}}$ have been determined to be $1.014 \pm 0.002$ and $0.974 \pm$ 0.002, respectively, based on a test set of forty electronic model chemistries[23].

One of the reviewers of this article pointed out that the $\alpha^{\mathrm{F} / \mathrm{ZPE}}$ value presented above also rationalizes very well the relation between the F and ZPE scaling factors obtained with DZP and TZP basis sets (which differ from those tested in ref. [23]) by the HF and MP2 methods as well as with DFT. The deviations are smaller than 1\% (see Tables 3 and 5 in Ref. [26] where the scaling factors are determined by fitting zero-point vibrational energies of 24 small molecules). A key point is that these relations reduce the problem to the calculation of only one parameternot three.

The ZPVE15/10 database is employed here to calculate $\lambda^{\mathrm{ZPE}}$. The $15 \mathrm{ZPEs}$ in this database are those for $\mathrm{C}_{2} \mathrm{H}_{2}, \mathrm{CH}_{4}, \mathrm{CO}_{2}, \mathrm{CO}, \mathrm{F}_{2}, \mathrm{H}_{2} \mathrm{CO}, \mathrm{H}_{2} \mathrm{O}, \mathrm{H}_{2}, \mathrm{HCN}, \mathrm{HF}, \mathrm{N}_{2} \mathrm{O}, \mathrm{N}_{2}, \mathrm{NH}_{3}, \mathrm{OH}$, and $\mathrm{Cl}_{2}$, and accurate values for these have been tabulated previously [23] and are given in Table 1. The optimal scale factor for ZPEs, $\lambda^{\mathrm{ZPE}}$, is obtained by minimizing the quantity

$$
\operatorname{RMSE}(\mathrm{ZPE})=\left\{\left[\sum_{m=1}^{15}\left(\lambda^{\mathrm{ZPE}} \varepsilon_{\mathrm{vib}, m}^{\mathrm{G}, \text { input }}-\varepsilon_{\mathrm{vib}, m}^{\mathrm{G}}\right)^{2}\right] / 15\right\}^{1 / 2}
$$

where $\varepsilon_{\mathrm{vib}, m}^{\mathrm{G}}$ is the ZPE of the $m^{\text {th }}$ molecule in the ZPE15 database, $\varepsilon_{\mathrm{vib}, m}^{\mathrm{G}, \text { input }}$ is the harmonic approximation to it obtained via

$$
\varepsilon_{\text {vib,m }}^{\mathrm{G}, \text { input }} \equiv \frac{h c}{2} \sum_{i} \omega_{i}
$$

where $\omega_{i}$ a computed harmonic frequencies of the molecule in units of $\mathrm{cm}^{-1}, h$ is Planck's constant, and $c$ is the speed of light. The value of $\lambda^{\mathrm{ZPE}}$ which minimizes RMSE(ZPE) in eqn 3 is obtained analytically from the expression

$$
\lambda^{\mathrm{ZPE}}=\frac{\sum_{m=1}^{15}\left(\varepsilon_{\mathrm{vib}, m}^{\mathrm{G}, \text { input }} \varepsilon_{\mathrm{vib}, m}^{\mathrm{G}}\right)}{\sum_{m=1}^{15}\left(\varepsilon_{\mathrm{vib}, m}^{\mathrm{G}, \text { input }}\right)^{2}}
$$

In order to save additional computational cost (which may be a consideration if one wishes to 
find the scale factor for an expensive, high-level method), we also developed a model called the "Reduced Scale Factor Optimization Model”, in which a subset from the original ZPE15 database of just 6 molecules is utilized in the optimization of $\lambda^{\mathrm{ZPE}}$ instead of the full set of 15 molecules comprising ZPE15. The six molecules used in the Reduced Scale Factor Optimization Model are $\mathrm{C}_{2} \mathrm{H}_{2}, \mathrm{CH}_{4}, \mathrm{H}_{2} \mathrm{CO}, \mathrm{H}_{2} \mathrm{O}, \mathrm{N}_{2} \mathrm{O}$, and $\mathrm{NH}_{3}$. This subset of molecules was selected based on the finding that the mean absolute deviation between the $\lambda^{\mathrm{ZPE}}$ values obtained from these molecules and the $\lambda^{\mathrm{ZPE}}$ values obtained from the full set of 15 molecules was only 0.0007 for the 40 electronic model chemistries tested. [23]

\section{Program Descriptions}

The FREQ program is made up of two basic components: (1) a Python program called Freqscale.py that calculates and outputs the optimal values for $\lambda^{\mathrm{ZPE}}, \lambda^{\mathrm{H}}$, and $\lambda^{\mathrm{F}}$ from a set of user-provided harmonic ZPEs, and (2), the Python scripts called input.py and pbs,py that instead allow the user to generate Gaussian 09 or Gaussian 03 input files and pbs files that calculate the ZPEs and provide them to Freqscale.py as a when the user cannot provide the harmonic frequencies a priori. Once the ZPEs are obtained by using the input.py and pbs,py, the final step of the calculation of $\lambda^{\mathrm{ZPE}}, \lambda^{\mathrm{H}}$, and $\lambda^{\mathrm{F}}$ is carried out by Freqscale.py. In the following two sections (3.1 and 3.2), we give detailed descriptions of these programs.

\subsection{Freqscale.py}

Freqscale.py is a Python program that computes the scale factors for zero-point energies, harmonic frequencies, and fundamental frequencies. The operation of this program requires an input file listing the computed ZPEs for the 15 molecules in Table 1, in units of $\mathrm{kcal} / \mathrm{mol}$, in the order: $\mathrm{C}_{2} \mathrm{H}_{2}, \mathrm{CH}_{4}, \mathrm{CO}_{2}, \mathrm{CO}, \mathrm{F}_{2}, \mathrm{H}_{2} \mathrm{CO}, \mathrm{H}_{2} \mathrm{O}, \mathrm{H}_{2}, \mathrm{HCN}, \mathrm{HF}, \mathrm{N}_{2} \mathrm{O}, \mathrm{N}_{2}, \mathrm{NH}_{3}, \mathrm{OH}$, and $\mathrm{Cl}_{2}$. We call this input file FREQ_com.txt, which will be generated by running the following two programs “input.py" and "pbs.py". Once these data are provided, the program calculates $\lambda^{\mathrm{ZPE}}, \lambda^{\mathrm{H}}$, and $\lambda^{\mathrm{F}}$ from eqns 5,1 , and 2 , respectively, and it prints the values for these scale factors to the screen. The code is provided below:

\section{Freqscale.py}

\# This is a program for computing various scale factors based on \# a scale factor optimization model. 


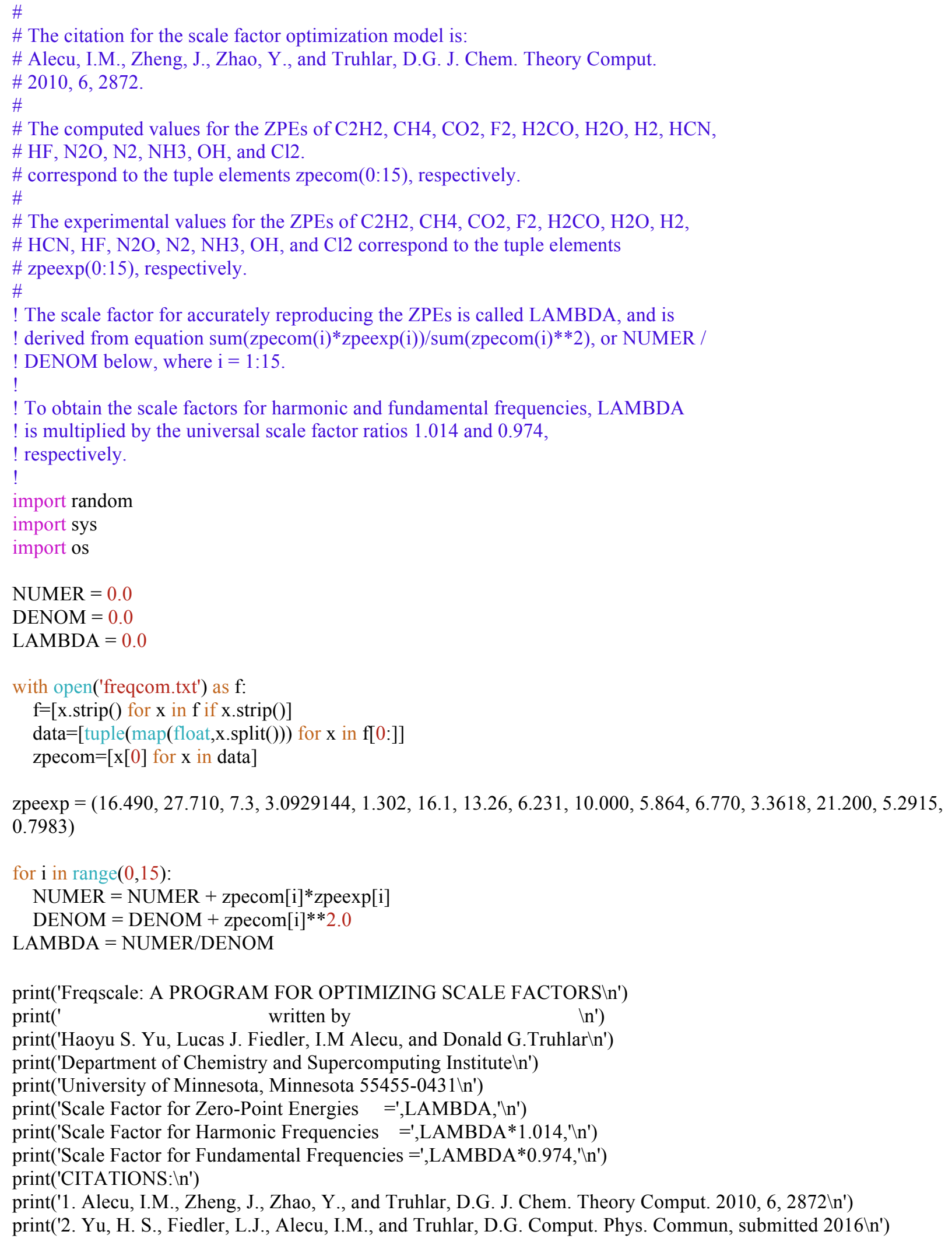




\section{2. input.py and pbs.py}

The input.py and pbs.py scripts allow the user to directly obtain Gaussian 03[27] or Gaussian 09[28] input files for computing the frequencies of $\mathrm{C}_{2} \mathrm{H}_{2}, \mathrm{CH}_{4}, \mathrm{CO}_{2}, \mathrm{CO}, \mathrm{F}_{2}, \mathrm{H}_{2} \mathrm{CO}$, $\mathrm{H}_{2} \mathrm{O}, \mathrm{H}_{2}, \mathrm{HCN}, \mathrm{HF}, \mathrm{N}_{2} \mathrm{O}, \mathrm{N}_{2}, \mathrm{NH}_{3}, \mathrm{OH}$, and $\mathrm{Cl}_{2}$ with the specified electronic model chemistry, and it submits these files to a queuing system. Once the frequency calculations have finished, the run.sh script will automatically grep the ZPEs listed in the Gaussian 09 output files into freqcom.txt file which is used to run Freqscale.py program.

The full operation of input.py is summarized by the flow chart in Figure 1. The python program will ask the user three questions, namely, "Which method are you going to choose?", then "Which basis set are you going to choose?", and lastly "What is the path of your basis set?" As we can see from Figure 1, there are two choices when you choose the basis set. Here are two examples, one for using a basis set built into Gaussian 09 and one for a user-defined basis set:

1. For a built-in basis set and the BLYP[29,30] functional as an example:

a. Which method are you going to choose? "BLYP"

b. Which basis set are you going to choose? "6-311G"

c. What is the path of your basis set? Hit the enter key

2. For a user-defined basis set and BLYP[29] functional as an example:

a. Which method are you going to choose? "BLYP"

b. Which basis set are you going to choose? "gen"

c. What is the path of your basis set? "home/truhlar/user1/basis_set/MG3S.gbs"

In the above examples, the calculation of the harmonic frequencies of $\mathrm{C}_{2} \mathrm{H}_{2}, \mathrm{CH}_{4}, \mathrm{CO}_{2}$, $\mathrm{CO}, \mathrm{F}_{2}, \mathrm{H}_{2} \mathrm{CO}, \mathrm{H}_{2} \mathrm{O}, \mathrm{H}_{2}, \mathrm{HCN}, \mathrm{HF}, \mathrm{N}_{2} \mathrm{O}, \mathrm{N}_{2}, \mathrm{NH}_{3}, \mathrm{OH}$, and $\mathrm{Cl}_{2}$ are using the BLYP[29] density functional paired with a particular basis set. If the basis set to be used is one that is already available in Gaussian 09, e.g., 6-311G[31,32], then the user can just type the keyword of this basis set. If the basis set to be used is not available in Gaussian 09, e.g., MG3S, the user can type "gen" and then the path of the external basis set. In addition, in the example above, all the other keywords for calculating the ZPEs of these 15 molecules are provided by input.py and pbs.py. The codes of these two programs are provided in the CPC Program Library. In the following two pages, we are going to show a representative part of the code, which is the creation of input and PBS files of $\mathrm{C}_{2} \mathrm{H}_{2}$ molecule. 


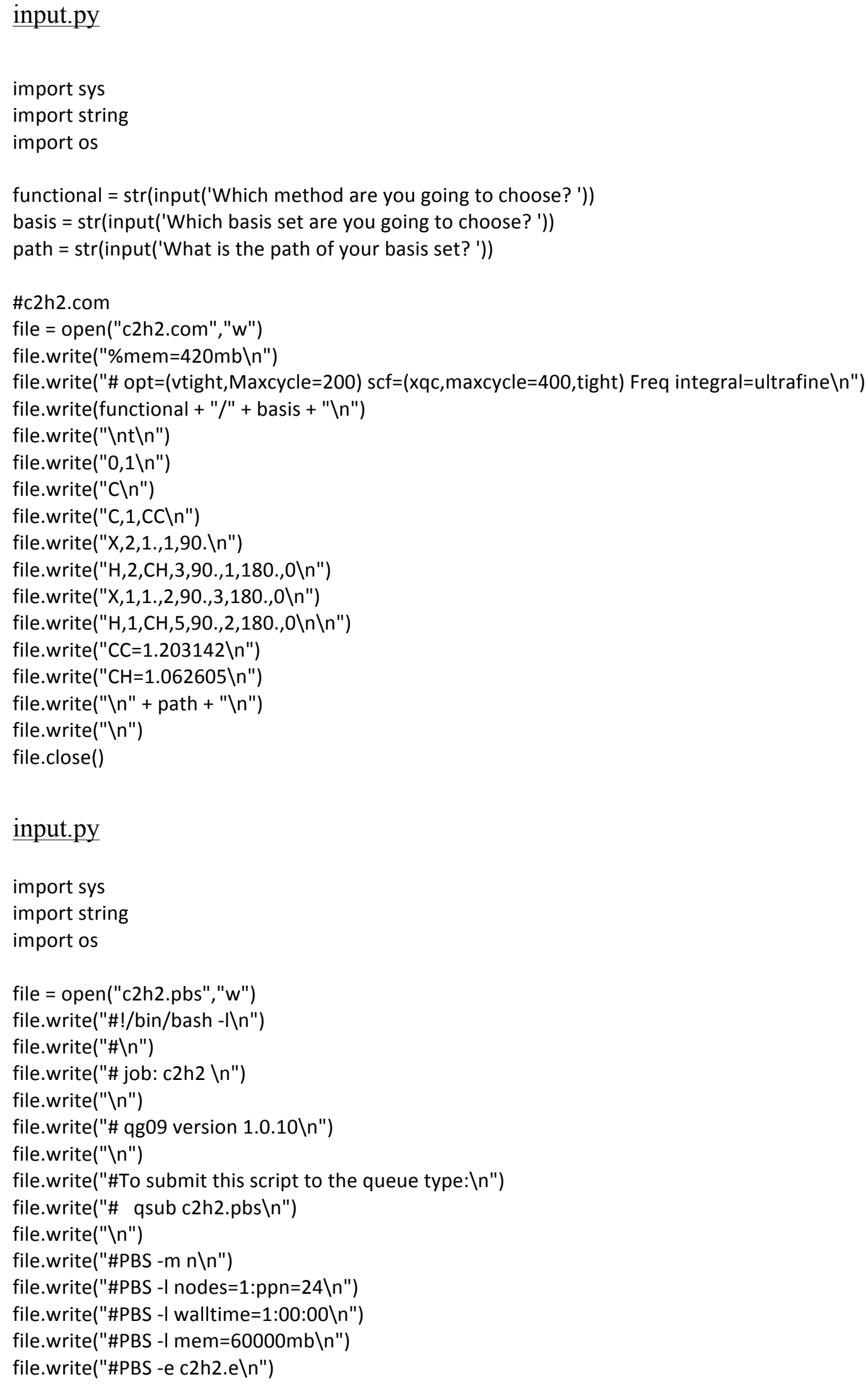


file.write("\#PBS -e c2h2.o\n")

file.write("\#PBS -q mesabi(n")

file.write("\#\n")

file.write("\n")

file.write("cd \$PBS_O_WORKDIR\n")

file.write("\n")

file.write("module load gaussian/g09.d01 \n")

file.write("/usr/bin/time g09 < c2h2.com >\& c2h2.out \n")

file.write("\n")

file.close()

\section{Discussion}

A few cautionary remarks are in order. First, because zero point energies are dominated by the higher frequencies, the scale factors determined by this method are especially appropriate for larger frequencies and for the whole zero-point energy; they may not be appropriate for, for example, low-frequency torsions or low-frequency bends. Second, since empirical scale factors are based on "normal" molecules they might be inappropriate for a molecule with an unusual bonding pattern. Third, the quasiharmonic approximation with scaled frequencies can be accurate for a molecule with a single conformation or for a single conformation of a molecule with multiple conformations if the barriers between conformational minima are high compared to the available thermal energy, but they do not treat multiple-structure contributions to partition functions. Other methods are available for multiple-structure anharmonicity [33,34,35,36], but the scaled vibrational frequencies determined the computer program presented here may be used to obtain vibrational frequencies to be used as input for the multiple-structure treatment, especially for the higher-frequency modes [37].

\section{Concluding Remarks}

Empirically scaling computed harmonic frequencies using appropriate scale factors is a widely employed and practical method for improving vibrational frequencies and zero-point energies from electronic structure calcuations. Here we provide a program, Freqscale.py that automates the scale factor optimization process. In the current version of FREQ, vibrational harmonic frequencies can be obtained by using the Gaussian 09 or Gaussian 03 input and pbs files provided by two python programs, input.py and pbs.py. 


\section{Computer program}

The computer program distribution is a tar file (FREQ.tar.gz) that contains five PYTHON programs, a bash script, and a manual, namely, Freqscale,py, run.sh, input.py, input6.py, pbs.py, pbs6.py, and Freqscale_Manual.pdf. An Excel worksheet is also provided, which can be used as an alternative way of obtaining the scale factors for ZPEs, fundamental frequencies, and harmonic frequencies. This material is available free of charge via the Internet at Computer Physics Communications Program Library (http://www.cpc.cs.qub.ac.uk).

\section{Acknowledgments}

The authors are grateful to the CPC Program Librarian, Carol Phillips, for suggestions that improved the program. This material is based on work supported by the U. S. Department of Energy, Office of Basic Energy Sciences, under grant no. DE-SC0008666. 
Table 1. The ZPE15 database: experimental ZPEs for the 15 molecules used in this work.

\begin{tabular}{ll}
\hline Molecule & ZPE (kcal/mol) \\
\hline $\mathrm{C}_{2} \mathrm{H}_{2}$ & $16.490^{a}$ \\
$\mathrm{CH}_{4}$ & $27.710^{a}$ \\
$\mathrm{CO}_{2}$ & $7.3^{a}$ \\
$\mathrm{CO}$ & $3.0929144^{a}$ \\
$\mathrm{~F}_{2}$ & $1.302^{a}$ \\
$\mathrm{H}_{2} \mathrm{CO}$ & $16.1^{a}$ \\
$\mathrm{H}_{2} \mathrm{O}$ & $13.26^{a}$ \\
$\mathrm{H}_{2}$ & $6.231^{a}$ \\
$\mathrm{HCN}$ & $10.000^{a}$ \\
$\mathrm{HF}$ & $5.864^{a}$ \\
$\mathrm{~N}_{2} \mathrm{O}$ & $6.770^{b}$ \\
$\mathrm{~N}_{2}$ & $3.3618^{a}$ \\
$\mathrm{NH}_{3}$ & $21.200^{c}$ \\
$\mathrm{OH}$ & $5.2915^{a}$ \\
$\mathrm{Cl}_{2}$ & $0.7983^{a}$ \\
\hline \multicolumn{3}{c}{${ }^{a}$ Irikura et al [38]. } \\
\multicolumn{3}{c}{ Grev et al [39]. } \\
\multicolumn{2}{c}{ Martin $[40]}$.
\end{tabular}




\section{Type the keyword of the basis set}

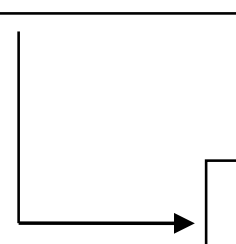

Create and submit Gaussian input files: $\mathrm{C}_{2} \mathrm{H}_{2}, \mathrm{CH}_{4}, \mathrm{CO}_{2}, \mathrm{CO}, \mathrm{F}_{2}, \mathrm{H}_{2} \mathrm{CO}, \mathrm{H}_{2} \mathrm{O}$, $\mathrm{H}_{2}, \mathrm{HCN}, \mathrm{HF}, \mathrm{N}_{2} \mathrm{O}, \mathrm{N}_{2}, \mathrm{NH}_{3}, \mathrm{OH}$, and $\mathrm{Cl}_{2}$

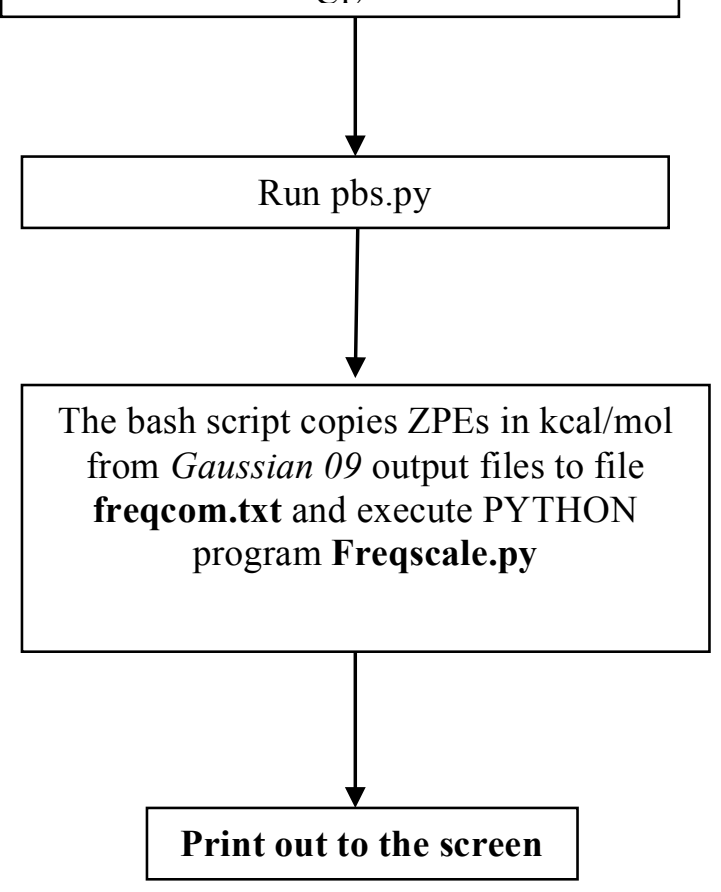

Figure 1: Flow chart describing the operation of the run.sh bash script. 


\section{References}

[1] J.A. Pople, Rev. Mol. Phys. 71 (1999) 1267.

[2] H. H. Nielsen, Encycl. Phys. 37 (1959) 173.

[3] I. M. Mills, In Molecular Spectroscopy: Modern Research; edited by K. N. Rao and C. W. Mathews; Academic: New York, 1972; pp. 115-140.

[4] Q. Zhang, P.N. Day, D.G. Truhlar, J. Chem. Phys. 98 (1993) 4948.

[5] K.M. Kuhler, D.G. Truhlar, A.D. Isaacson, J. Chem. Phys. 104 (1996) 4664.

[6] A.D. Isaacson, J. Chem. Phys. 108 (1998) 9978.

[7] V. Barone, J. Chem. Phys. 122 (2005) 014108.

[8] J. Bloino, M. Biczyzko, V. Barone, J. Chem. Theory Comput. 8 (2012) 1015.

[9] R. J. Whitehead, N. C. Handy, J. Mol. Spectrosc. 55 (1975) 356.

[10] S. Carter, N. C. Handy, Phys. Rep. 5 (1986) 117.

[11] S. C. Tucker, T. C. Thompson, J. G. Lauderdale, and D. G. Truhlar, Comput. Phys. Commun. 51, 233 (1988).

[12] B. T. Sutcliffe, S. Miller, J. Tennyson, Comput. Phys. Commun. 51 (1988) 73.

[13] D. H. Zhang, J. Z. H. Zhang, J. Chem. Phys. 99 (1993) 6624.

[14] S. Carter, J. M. Bowman, N. C. Handy, Theor. Chem. Acc. 100 (1998) 191.

[15] M. Mladenovic, M. Lewerenz, Chem. Phys. Lett. 321 (2000) 135.

[16] Y. Volobuev, W. C. Necoechea, D. G. Truhlar, Chem. Phys. Lett. 330 (2000) 471.

[17] D.P. Tew, N.C. Handy, S. Carter, Phys. Chem. Chem. Phys. 3 (2001) 1958.

[18] A. Chakraborty, D. G. Truhlar, J. M. Bowman, S. Carter, J. Chem. Phys. 121 (2004) 2071.

[19] S. L. Mielke, A. Chakraborty, D. G. Truhlar, J. Phys. Chem. A 117 (2013) 7327.

[20] R.D. Johnson III, K.K. Irikura, R.N. Kacker, R. Kessel, J. Chem. Theory Comput. 6 (2010) 2822.

[21] J.A. Pople, A.P. Scott, M.W. Wong, L. Radom, Isr. J. Chem. 33 (1993) 345.

[22] A.P. Scott, L. Radom, J. Phys. Chem. 100 (1996) 16502.

[23] I.M. Alecu, J. Zheng, Y. Zhao, D.G. Truhlar, J. Chem. Theory Comput. 6 (2010) 2872.

[24] Y. Zhao, D.G. Truhlar, Theor. Chem. Acc. 120 (2008) 215.

[25] P.L. Fast, J. Corchado, M.L. Sanchez, D.G. Truhlar, J. Phys. Chem. A 103 (1999) 3139.

[26] S. G. Andrade, L. C. S. Goncalves, and D. E. Jorge, J. Mol. Struct. 864 (2008) 20.

[27] M.J. Frisch, G.W. Trucks, H.B. Schlegel, G.E. Scuseria, M.A. Robb, J.R. Cheeseman, J.A.J. Montgomery, T. Vreven, K.N. Kudin, J.C. Burant, J.M. Millam, S.S. Iyengar, J. Tomasi, V. Barone, B. Mennucci, M. Cossi, G. Scalmani, N. Rega, G.A. Petersson, H. Nakatsuji, M. 
Hada, M. Ehara, K. Toyota, R. Fukuda, J. Hasegawa, M. Ishida, T. Nakajima, Y. Honda, O. Kitao, H. Nakai, M. Klene, X. Li, J.E. Knox, H.P. Hratchian, J.B. Cross, V. Bakken, C. Adamo, J. Jaramillo, R. Gomperts, R.E. Stratmann, O. Yazyev, A.J. Austin, R. Cammi, C. Pomelli, J.W. Ochterski, P.Y. Ayala, K. Morokuma, G.A. Voth, P. Salvador, J.J. Dannenberg, V.G. Zakrzewski, S. Dapprich, A.D. Daniels, M.C. Strain, O. Farkas, D.K. Malick, A.D. Rabuck, K. Raghavachari, J.B. Foresman, J.V. Ortiz, Q. Cui, A.G. Baboul, S. Clifford, J. Cioslowski, B.B. Stefanov, G. Liu, A. Liashenko, P. Piskorz, I. Komaromi, R.L. Martin, D.J. Fox, T. Keith, A.-L.M. A, C.Y. Peng, A. Nanayakkara, M. Challacombe, P.M.W. Gill, B. Johnson, W. Chen, M.W. Wong, C. Gonzalez, J.A. Pople, Gaussian 03, revision E.01; Gaussian, Inc., (Wallingford, CT, 2004).

[28] M.J. Frisch, G.W. Trucks, H.B. Schlegel, G.E. Scuseria, M.A. Robb, J.R. Cheeseman, G. Scalmani, V. Barone, B. Mennucci, G.A. Petersson, H. Nakatsuji, M. Caricato, X. Li, H.P. Hratchian, A.F. Izmaylov, J. Bloino, G. Zheng, J.L. Sonnenberg, M. Hada, M. Ehara, K. Toyota, R. Fukuda, J. Hasegawa, M. Ishida, T. Nakajima, Y. Honda, O. Kitao, H. Nakai, T. Vreven, J.A.J. Montgomery, J.E. Peralta, F. Ogliaro, M. Bearpark, J.J. Heyd, E. Brothers, K.N. Kudin, V.N. Staroverov, R. Kobayashi, J. Normand, K. Raghavachari, A. Rendell, J.C. Burant, S.S. Iyengar, J. Tomasi, M. Cossi, N. Rega, J.M. Millam, M. Klene, J.E. Knox, J.B. Cross, V. Bakken, C. Adamo, J. Jaramillo, R. Gomperts, R.E. Stratmann, O. Yazyev, A.J. Austin, R. Cammi, C. Pomelli, J.W. Ochterski, R.L. Martin, K. Morokuma, V.G. Zakrzewski, G.A. Voth, P. Salvador, J.J. Dannenberg, S. Dapprich, A.D. Daniels, O. Farkas, J.B. Foresman, J.V. Ortiz, J. Cioslowski, D.J. Fox, Gaussian 09, revision A.02; Gaussian, Inc., (Wallingford, CT, 2009).

[29] A. D. Becke, Phys. Rev. A 38 (1988) 3098.

[30] C. Lee, W. Yang, R. G. Parr, Phys. Rev. B 37 (1988) 785.

[31] R. Krishnan, J. S. Binkley, R. Seeger, J. A. Pople, J. Chem. Phys. 72 (1980) 650.

[32] A. D. McLean, G. S. Chandler, J. Chem. Phys. 72 (1980) 5639.

[33] J. Zheng, T. Yu, E. Papajak, I, M. Alecu, S. L. Mielke, and D. G. Truhlar, Physical Chemistry Chemical Physics 13 (2011) 10885.

[34] J. Zheng, S. L Mielke, K. L Clarkson, D. G. Truhlar, Comput. Phys. Commun. 183 (2012) 1803.

[35] J. Zheng, D. G. Truhlar, J. Chem. Theory Comput. 9 (2013) 1356.

[36] J. Zheng, R. Meana-Pañeda, and D. G. Truhlar, Comput. Phys. Commun. 184 (2013) 2032.

[37] J. Zheng, R. Meana-Pañeda, and D. G. Truhlar, J. Amer. Chem. Soc. 136 (2014) 5150.

[38] K.K. Irikura, J. Phys. Chem. Ref. Data, 36 (2007) 389.

[39] R.S. Grev, C.L. Janssen, H.F. Schaefer III, J. Chem. Phys. 95 (1991) 5128.

[40] J.M.L. Martin, J. Chem. Phys. 97 (1992) 5012. 